\title{
Correction to: Simple motion correction strategy reduces respiratory-induced motion artifacts for k-t accelerated and compressed-sensing cardiovascular magnetic resonance perfusion imaging
}

Ruixi Zhou ${ }^{1,2 \dagger}$, Wei Huang ${ }^{1 \dagger}$, Yang Yang ${ }^{1,2}$, Xiao Chen ${ }^{3}$, Daniel S. Weller ${ }^{4}$, Christopher M. Kramer ${ }^{1,5}$, Sebastian Kozerke ${ }^{6}$ and Michael Salerno 1,2,5*

\section{Correction}

Figure 1 of this original publication [1] contained a minor error as one of the lines in the "Reconstruction pipline" was not visible. The updated Fig. 1 is published in this correction article.

\begin{abstract}
Author details
'Department of Medicine, University of Virginia Health System,

Charlottesville, VA, USA. ${ }^{2}$ Department of Biomedical Engineering, University of Virginia Health System, Charlottesville, VA, USA. ${ }^{3}$ Medical Imaging Technologies, Siemens Healthineers, Princeton, NJ, USA. ${ }^{4}$ Department of Electrical and Computer Engineering, University of Virginia, Charlottesville, VA, USA. ${ }^{5}$ Department of Radiology and Medical Imaging, University of Virginia Health System, Charlottesville, VA, USA. ${ }^{6}$ Department of Information Technology and Electrical Engineering, Institute for Biomedical Engineering, University and ETH Zurich, Zurich, Switzerland.
\end{abstract}

Received: 7 February 2018 Accepted: 19 February 2018

Published online: 26 March 2018

\section{Reference}

1. Zhou R, Huang W, Yang $Y$, et al. Simple motion correction strategy reduces respiratory-induced motion artifacts for k-t accelerated and compressedsensing cardiovascular magnetic resonance perfusion imaging. J Cardiovasc Magn Reson. 2018;20:6. https://doi.org/10.1186/s12968-018-0427-1.

\footnotetext{
* Correspondence: ms5pc@virginia.edu

${ }^{\dagger}$ Equal contributors

'Department of Medicine, University of Virginia Health System,

Charlottesville, VA, USA

${ }^{2}$ Department of Biomedical Engineering, University of Virginia Health System,

Charlottesville, VA, USA
} 


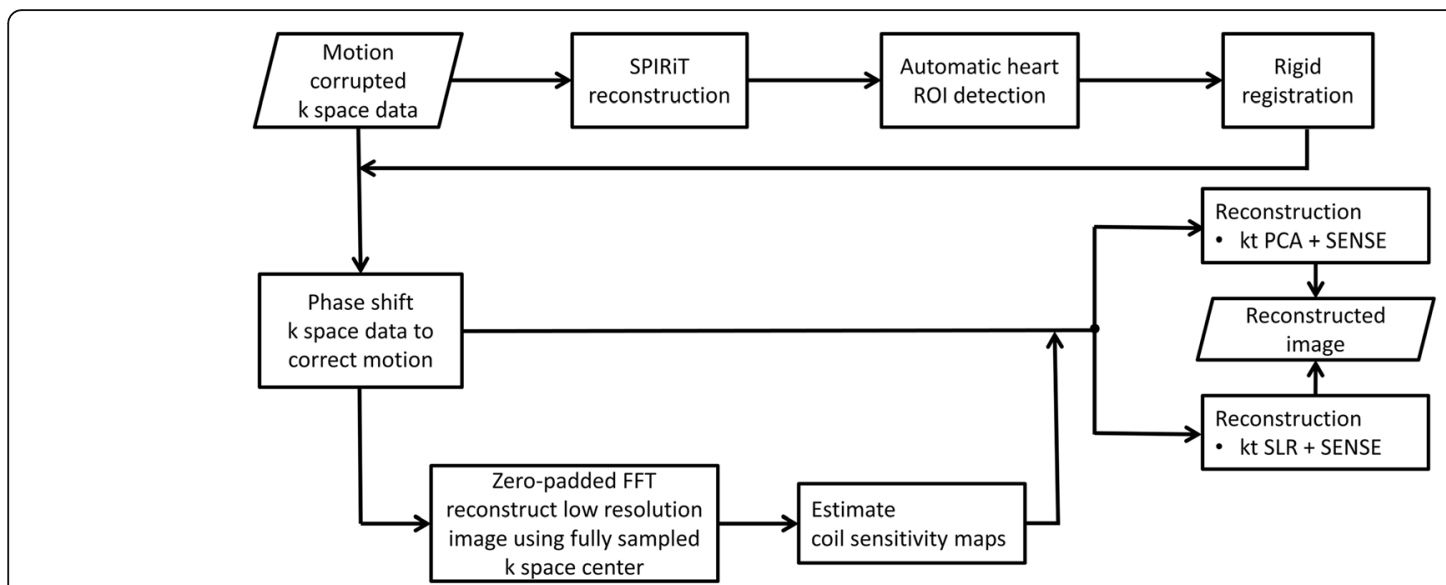

Fig. 1 Corrected Reconstruction pipeline 